Meta

Journal des traducteurs

Translators' Journal

\title{
Langue métissée et traduction : quelques enjeux théoriques
}

\section{Rohan Anthony Lewis}

Volume 48, numéro 3, septembre 2003

Traduction et enseignement

Translation and teaching

URI : https://id.erudit.org/iderudit/007601ar

DOI : https://doi.org/10.7202/007601ar

Aller au sommaire du numéro

Éditeur(s)

Les Presses de l'Université de Montréal

ISSN

0026-0452 (imprimé)

1492-1421 (numérique)

Découvrir la revue

Citer cet article

Anthony Lewis, R. (2003). Langue métissée et traduction : quelques enjeux théoriques. Meta, 48(3), 411-420. https://doi.org/10.7202/007601ar

\section{Résumé de l'article}

Bien que l'on soit en peine de trouver une homogénéité à l'intérieur de ce qu'il est convenu de considérer comme une seule et même langue, la théorisation et l'enseignement de la traduction se fondent par convention sur l'idée que la langue est une entité relativement stable, aux frontières bien définies. Cet article, de caractère exploratoire, cherche à expliciter le contexte historique dont est issue la notion conventionnelle de langue utilisée en traduction, ce qui amène à se demander comment une conception de la langue qui reconnaîtrait le métissage linguistique pourrait modifier notre conception de la traduction et de son enseignement.
Ce document est protégé par la loi sur le droit d'auteur. L'utilisation des services d’Érudit (y compris la reproduction) est assujettie à sa politique d'utilisation que vous pouvez consulter en ligne.

https://apropos.erudit.org/fr/usagers/politique-dutilisation/ 


\title{
Langue métissée et traduction: quelques enjeux théoriques
}

\author{
ROHAN ANTHONY LEWIS \\ Université de Montréal, Montréal, Canada \\ roanthony@yahoo.com
}

\begin{abstract}
RÉSUMÉ
Bien que l'on soit en peine de trouver une homogénéité à l'intérieur de ce qu'il est convenu de considérer comme une seule et même langue, la théorisation et l'enseignement de la traduction se fondent par convention sur l'idée que la langue est une entité relativement stable, aux frontières bien définies. Cet article, de caractère exploratoire, cherche à expliciter le contexte historique dont est issue la notion conventionnelle de langue utilisée en traduction, ce qui amène à se demander comment une conception de la langue qui reconnaîtrait le métissage linguistique pourrait modifier notre conception de la traduction et de son enseignement.
\end{abstract}

\section{ABSTRACT}

Despite the fact that it is difficult to identify any kind of homogeneity within the confines of what is conveniently referred to as one and the same language, translation theorisation and teaching have traditionally been based on the idea that language is a stable entity with relatively well defined frontiers. This exploratory article seeks to locate the historical context that gave rise to this orthodox notion of language used in translation as well as ask how a conception of language that considers linguistic "mixedness" could modify our conception of translation and its teaching.

\section{MOTS-CLÉS/KEYWORDS}

théorisation de la traduction, enseignement de la traduction, langue métissée, hybridation linguistique

\section{Langue et théorisation de la traduction}

Le contexte postcolonial et postmoderne met en évidence des variétés linguistiques qui, de par leur manque d'homogénéité ou de stabilité, s'inscrivent difficilement dans le cadre de ce qu'on qualifie conventionnellement comme étant des «langues». Nées de processus de métissage horizontal ou vertical, ces variétés mixtes et non standard comprennent le plus souvent des multiplicités de «lectes» provenant de plusieurs langues.

L'interpénétration horizontale s'entend du métissage de deux ou de plusieurs langues qui fait s'effondrer les frontières fixes séparant celles-ci. Le spanglish frontalier des États-Unis et du Mexique est un cas classique de ce type de métissage. L'interpénétration verticale correspond à la manifestation, à l'intérieur d'une même communauté linguistique, de plusieurs couches dialectales, certaines tendant à se rapprocher d'un pôle standard et d'autres tendant à s'écarter de ce même pôle, comme dans le cas des présumés continuums créoles de plusieurs pays des Caraïbes tels que le Belize, la Guyana et la Jamaïque.

Meta, XLVIII, 3, 2003 
Il faut cependant signaler que toutes les variétés linguistiques participent à ce genre de métissage. Les sociolinguistes s'accordent pour dire que, très souvent, les dialectes qu'on dénomme «langues», y compris les langues standard, se mélangent les uns aux autres, formant des continuums qui transcendent les frontières tant géographiques que politiques et sociales, pour constituer des amalgames linguistiques. Or, les professions langagières, telle la traduction, ne tiennent guère compte de cette fluidité, prétendant que ce qu'on appelle «langue» doit être forcément une variété relativement stable ou uniformisée. De ce point de vue, toute manifestation d'hétérogénéité linguistique est perçue comme une aberration. Cette conception de la langue correspond à la notion saussurienne de «système» linguistique ou à la langue d'une communauté homogène de locuteurs et d'écouteurs idéaux telle que conçue par Chomsky (1965: 3).

Si les langues métissées revêtent une importance relative et croissante aujourd'hui, cela est principalement attribuable à la migration ainsi qu'à la revendication culturelle de certaines communautés qui perçoivent ces variétés comme faisant partie intégrante de leur identité. Pour cette raison, il n'est pas surprenant qu'elles entrent de plus en plus dans le cadre de pratiques traduisantes qui se réalisent cependant en l'absence d'une réflexion adéquate sur les défis que posent ces variétés à l'égard des conceptions conventionnelles de la langue et de la traduction. D'où un «trou béant» du point de vue de la pédagogie de la traduction.

\section{Texte de départ, texte d'arrivée; langue de départ, langue d'arrivée}

En général, deux approches types du processus traduisant ont guidé les enseignants de la traduction. La première est axée sur la recherche de correspondances - non seulement correspondance textuelle, mais aussi linguistique - entre la langue de départ et la langue d'arrivée, tandis que la deuxième cherche à reproduire ou à réexprimer le «sens» de la langue de départ dans la langue d'arrivée. Cette dernière approche prétend mettre l'accent sur des variables culturelles et contextuelles plutôt que linguistiques. Ainsi peut-on constater que la première approche privilégie la recherche de correspondances comme étant la caractéristique qui conditionne l'opération traduisante alors que la deuxième met l'accent sur le sens derrière le signe linguistique ou au-delà de celui-ci.

La première approche a été particulièrement influente pendant la période d'après-guerre, quand la linguistique structuraliste jouait un rôle déterminant sur l'évolution de la traductologie. Comme la traduction était perçue comme une opération sur la langue, conçue comme la forme linguistique (la langue saussurienne) et non comme la fonction ou l'usage linguistique (la parole saussurienne) (Beaugrande 1994: 9), il semblait plus naturel que la linguistique structuraliste puisse répondre aux questions théoriques touchant la traduction. Celle-ci a donc été définie par rapport aux langues: langue de départ et langue d'arrivée. Or, cette définition relevait de la division structuraliste des langues en "systèmes», chacun se distinguant des autres.

Plus récemment, les théoriciens de la traduction, s'inspirant des tendances antistructuralistes, ont manifesté une certaine préférence pour les notions de «texte de départ» et de «texte d'arrivée», plutôt que pour les notions traditionnelles de «langue 
de départ» et «langue d'arrivée». Ils ont donc mis l'accent sur le transfert du sens entre textes, et non entre langues. Toutefois, à cause de la nécessité de distinguer entre la traduction et d'autres pratiques telles l'adaptation et la réécriture - deux pratiques qui ressemblent à la traduction, mais qui se font à l'intérieur d'une «même langue »- ils ont maintenu la différence entre la langue du texte de départ et celle du texte d'arrivée en traduction. La conception de la traduction comme pratique renvoyant à cette distinction interlinguistique - texte de la langue de départ et texte de la langue d'arrivée - reste donc prédominante en traduction. En d'autres mots, pour que le transfert du sens entre textes soit considéré comme de la traduction, il faut que ce transfert se passe entre des textes dans deux langues différentes. L'accent mis sur le transfert entre les textes en traduction n'a donc pas changé l'importance accordée au transfert entre langues. Autrement dit, quelle que soit l'approche théorique renforçant la pédagogie de la traduction, par traduction, on entend toujours un rapport entre textes rédigés en langue $A$ et traduits en langue B. On revient donc, implicitement, à des correspondances entre systèmes linguistiques distinctes et stables.

La définition de la traduction d'Al-Shabab, qui insiste sur le fait que la traduction consiste en l'interprétation d'un texte linguistique ou verbal dans une langue différente de celle dans laquelle il était écrit au départ, montre l'importance du lien entre texte de départ ou d'arrivée et langue de départ ou d'arrivée. Mais la dimension la plus importante de la définition fournie par Al-Shabab a trait à la langue en tant qu'objet de l'opération traduisante. En précisant ce qu'il entend par langue, il constate:

'Language' is not used here in the sense of a dialect; nor is it used in a metaphorical sense to mean the language of music or dance; nor is it used in the sense of artificial language such as a computer language [...] 'Language' is used here in the ordinary everyday meaning of the word. Namely, it is the tongue - verbal code - used by a human community, large or small, for instance English, Arabic, Aramaic, etc. (1996: 8)

Cette conception de la langue réunit deux notions différentes: celle d'une langue communautaire et celle d'une langue normalisée ou standardisée. Étant donné qu'Al-Shabab écarte les variétés dialectales de sa définition, il oppose celles-ci à la langue «ordinaire et communautaire». Mais si la langue «ordinaire et communautaire» ne correspond pas à des variétés dialectales, à quoi correspond-elle? Il semble, d'après Al-Shabab, qu'elle corresponde nécessairement à une langue standardisée. Cette notion renvoie à une conception de la langue comme étant une entité stable et homogène. Or, Pergnier fait remarquer qu'il est impossible «de trouver [...] une homogénéité à l'intérieur de ce qu'il est convenu de considérer une seule et même langue» (1993: 248).

Malgré le constat de Pergnier, on observe que les professeurs de traduction s'appuient sur la conception de langue telle qu'articulée par Al-Shabab, en faisant appel aux notions de langue de départ et de langue d'arrivée comme si celles-ci étaient des synonymes de texte de départ et de texte d'arrivée. Cette conception fonde la pratique traduisante sur la symétrie linguistique, symétrie qui n'est possible qu'entre les langues standard. Il faut donc se demander si la conception conventionnelle de la langue à laquelle on a recours en traduction rend compte de tous les contextes linguistiques pertinents à la traduction et si les notions qui en découlent, à savoir l'équivalence, la fidélité et le sens (voir les thèses de Seleskovitch, Lederer et Delisle), ont des assises théoriques solides. 
Certes, plusieurs chercheurs se sont interrogés sur la pertinence de notions telles que l'équivalence et la fidélité en traduction et ont même proposé qu'on les élimine du discours sur la traduction. Cependant, à de rares exceptions près, l'interrogation sur la pertinence de ces notions se limite à considérer leur usage en traduction (voir, par exemple, Pym 1992: 37-49) et le sens qui leur est donné dans ce contexte, et non le cadre historique et culturel dont ces notions sont issues. À partir d'une réflexion sur les langues métissées, nous envisageons les problèmes théoriques de la traduction et son enseignement, en mettant l'accent sur la conception historique de la langue à la base de la pratique. Par exemple, nous montrons que les problèmes théoriques touchant l'équivalence ne relèvent pas de l'usage de ce terme dans le discours sur la traduction mais de sa valeur en tant que produit de certains rapports historiques interlinguistiques.

\section{Langue distincte et traduction}

Pour plusieurs traductologues, la traduction dépend d'un rapport interlinguistique, relevant d'une "égalité» entre langue source et langue cible. Cette égalité, qui se situe au niveau de la forme, de la fonction ou de l'usage, n'existe que dans le cadre de variétés standardisées. Bien entendu, cette notion d'égalité entre langues est enracinée dans l'histoire linguistique de l'Occident, où le latin a servi de modèle pour les formes, les fonctions et les usages des langues en voie de standardisation ${ }^{1}$ tels le français, l'espagnol, etc. Cette histoire est donc la fondation à partir de laquelle s'est élaborée l'idée d'un parallélisme entre les langues, qui ferait qu'un concept ou un terme exprimé dans une langue aurait un homologue dans une autre langue et que ces langues seraient autonomes et distinctes les unes des autres.

Lorsque Wright (1997: 7) constate qu'on a traduit entre le latin et le roman, au sens normal du terme, seulement au XII ${ }^{\mathrm{e}}$ siècle, car le latin et le roman ne constituaient pas deux langues autonomes nécessaires à la traduction, il se situe précisément dans le cadre d'une conception des langues comme étant des entités séparées et autonomes que s'insèrent les pratiques diverses appelées traduction. Dans la perspective de Wright, la traduction dépendrait non seulement d'une distance ou d'une séparation entre deux variétés linguistiques, mais aussi de l'autonomie de celles-ci. La traduction serait alors une opération par laquelle un traducteur franchit la frontière séparant deux «langues» pour faire passer le sens et pour reproduire dans l'une (la langue d'arrivée) un texte qui serait fidèle et équivalent à celui de l'autre (la langue de départ). Il s'ensuit que cette conception de la traduction, qui représente en fait la pensée dominante en traductologie, repose sur l'idée qu'il existe une exclusivité mutuelle des langues, laquelle empêche la communication entre des communautés utilisant des langues différentes et les oblige à traduire.

Il importe ici de cerner plus précisément le rôle qu'a joué la standardisation dans l'évolution de la conception dominante de la traduction et ses conséquences pour l'enseignement de la traduction. L'histoire de la standardisation des langues, précédée de leur normalisation ${ }^{2}$, en passant par la Réforme protestante, remonte aux fondations de la civilisation gréco-romaine. Martin Luther, par exemple, qui a traduit les écritures chrétiennes à partir du latin standard pour le peuple allemand, s'est appuyé sur le modèle de standardisation légué par cette tradition pour formuler une norme allemande. Confronté au défi important de traduire le texte biblique d'une langue 
littéraire, homogène (le latin standard) en une langue instable, comprenant plusieurs dialectes (les dialectes allemands), Luther a dû intervenir dans l'histoire pour influencer l'évolution d'une langue. Il a eu recours à la grammaire latine pour «fixer » un dialecte littéraire allemand, lequel, selon Baggioni, a servi de norme pour la traduction de la Bible ainsi que pour la promotion de l'alphabétisation des populations de l'aire appelée aujourd'hui "germanophone» (1997: 88). En termes élogieux, Baggioni reconnaît la traduction biblique de Luther, ce «faiseur de langue», comme «l'action primordiale d'un individu sur la détermination de la norme d'une langue commune» (ibid.). Par son action de «faiseur de langue», Luther a établi une certaine symétrie entre la langue d'arrivée et la langue de départ, qu’il croyait essentielle à la traduction.

La traduction de Luther est importante pour deux raisons: elle a tout d'abord créé une variété linguistique normalisée, l'allemand, pour traduire la Bible mais c'est par le biais de la traduction que cette langue normalisée a été créée. On peut dire ainsi que la traduction requiert conventionnellement l'existence d'une langue normalisée et que la traduction peut normaliser la langue, révélant clairement la symbiose qui existe entre standardisation et traduction. Quoiqu'il soit possible de traiter ces deux éléments distinctement, leur conséquence sur la manière de concevoir la traduction reste la même: la pratique de la traduction repose sur l'existence préalable de la langue normalisée, et si celle-ci n'existe pas, elle la créera, du fait que les langues normalisées ou standardisées sont mieux placées pour entretenir des rapports d'égalité et de correspondance puisqu'elles sont relativement stables. Outre l'exemple de la traduction de la Réforme à laquelle participaient des traducteurs tel Luther, ce phénomène est perceptible de façon très évidente dans le contexte de la traduction coloniale.

Presque partout où le colonialisme européen a rencontré des situations linguistiques qui ne correspondaient pas à la notion dominante européenne de la langue, les Européens ont voulu imposer la leur. Ainsi des langues et des dialectes, principalement oraux et comprenant des continuums linguistiques, ont été stabilisés et homogénéisés selon le modèle européen, et cela, comme l'avait fait Luther, dans le but de faciliter la traduction. Dans le contexte colonial, pour que la traduction devienne possible, le colonisateur, habituellement un missionnaire, devait repérer un «lecte» (ou des éléments de plusieurs «lectes») considéré digne de constituer le standard de la nouvelle «langue» autochtone. Cette variété subissait alors une suppression de sa variabilité et une régularisation de sa grammaire. Aussi la traduisibilité des langues non européennes, tout comme celle des langues européennes avant elles, s'est-elle trouvée dépendante de la standardisation.

\section{Enjeux théoriques de la langue métissée}

Non seulement prétend-on que le contexte linguistique et culturel de la standardisation est reproductible partout, mais aussi qu'il est le seul qui puisse valablement sous-tendre une conception de la traduction. Or, comme nous l'avons mentionné au début de cet article, le contexte postmoderne ou postcolonial nous présente de plus en plus des contextes de langues métissées. Le plus souvent, ces langues ne subissent et ne subiront aucun processus de standardisation. Elles n'entretiennent donc pas de rapport d'égalité avec d'autres langues, ce qui pose, comme le souligne Mehrez, un problème théorique et pratique pour la traduction du point de vue de l'équivalence 
et de la fidélité d'un sens à transmettre (1992:121-122). Dans les pays postcoloniaux, par exemple, certains écrivains (dont Patrick Chamoiseau, Gabriel Okara, Sam Selvon, pour ne nommer que quelques-uns) ont essayé de faire entendre leur voix à travers des langues européennes qui leur avaient été imposées. Les textes qu'ils ont produits sont devenus « a combined version of other literary by-products resulting from an indigenous speech pattern, thinking patterns and world view [...] transliterated into the European language» (Ojo, 1986: 295). En passant leur texte par la matrice de leurs propres cultures (1986: 294), ces écrivains finissent par tisser un texte dont le contenu, comme le fait remarquer Mehrez, est le produit de plus d'une culture, plus d'une langue ou plus d'une expérience du monde (1992: 122). Tout en reconnaissant qu'un tel texte pourrait avoir une influence déterminante sur notre conception de la traduction, Mehrez souligne que, de par sa fluidité linguistique, ce texte effacerait la distinction traditionnelle entre langue de départ et langue d'arrivée. Un texte rédigé dans cette «langue» n'appartiendrait donc plus à une «langue» en tant que telle, l'interpénétration de plusieurs langues dans le même texte ayant abouti à sa transformation en langue «hybride» ou «métissée».

Examinons comme exemple de cette variété métissée un extrait d'un article de presse, écrit par le journaliste jamaïquain C. Roy Reynolds.

Dem seh dankey noh business ena cow fight far him don't ave horn.

Soh mi noh business ena a hintellectual fight since di ongly time dem dat mi go ah igh school is fi goh cova tings fi newspaper. But den again dem seh fools rush in whey angel fraid fe trad, an soh mi really ave a licence fi rush in to.

Soh jack mandora mi noh really choose none but di agument between di two siid meck mi rememba a story ah hear when mi did ah goh a Faam School.

Meck sure yuh undastan mi, far mi noh seh mi did ah goh a famitory school, mi seh Faam school. Eny way accadin to di story mi did a cum fram maakit wan day an big, big agument staat between di ooman dem bout a wah a di baddis pain.

Wan ooman seh is headache, nedda wan seh is baby bawning pain, an de odda wan seh is teethache. Well, dem chat, chat, chat but dem cuddn't cum to eny understanding. Soh dem tun to di man weh nevah bisniss wid di agument soh far an ask im fi umpiah it. Di man schups him mout an den him tell dem: Di ole ah unno ah taak piss. Eny ah unno eva mash unno balls? Lawd ah massy story dun, far dat deh is di ongly pain I kno whey can stap yu breat ${ }^{3}$.

\section{[TRADUCTION EN ANGLAIS ${ }^{4}$ :}

It is said that the donkey should stay out of cow fights since he has no horns.

I should, therefore, not get involved in a fight between intellectuals, since the only times I set foot in high schools were to cover stories for the newspaper. But then again, they say fools rush in where angels fear to tread, so that gives me licence to rush in.

Well, let it be understood that I am not partial to either side; but the argument between the two reminds me of a story I once heard while attending Form school.

Get it clear: I did not say while attending the Reformatory, I said while attending Form school. Anyway, as the story goes, I was returning from the market one day and witnessed the beginnings of a very heated discussion between some market women about what the most intense pain was.

One woman said it was a headache, another said it was childbirth, and the third said it was a toothache. Well, they continued talking but could come to no agreement. They turned to a man, who, up to that point, had stayed clear of the argument, and asked him to umpire it for them. The man hissed his teeth and said to them: "You're all 
talking garbage. Has any of you ever been hit in the balls? End of story! My goodness! That is the only pain I know that can make you stop breathing!]

Ce texte comprend plusieurs éléments basilectaux du continuum jamaïquain, bien que l'auteur fasse usage d'éléments mésolectaux et acrolectaux ${ }^{5}$. Le mot don't dans l'expression "him don't ave horn», selon sa prononciation, appartient soit au mésolecte, soit à l'acrolecte. Le mot «fools» dans la phrase «dem seh fools rush in» respecte les règles morphologiques de pluralisation de l'acrolecte, mais fait partie également du mésolecte. Le basilecte ne nécessiterait pas ce genre d'inclinaison pour marquer le pluriel puisqu'il est clair dans le contexte. D'autre part, «di agument between di two siid» respecte les règles de pluralisation du basilecte puisque l'adjectif a déjà signalé le fait qu'on parle d'une quantité plurielle. L'adjectif redoublé big, big dans «big, big agument staat», qui porte sur l'intensité de la discussion, est un exemple d'une morphologie africaine gouvernant des lexèmes européens. Par contre, la phrase "Wan ooman seh is headache, nedda wan seh is baby bawning pain, an de odda wan seh is teethache» fait usage d'un élément acrolectal, à savoir, le verbe «is». Cependant, ce « is» n'est que le remplacement de la particule « $a$ » qui traduit l'anglais: «[it] is/was» comme dans le fragment de phrase «wah $a$ di baddis pain». Le mot «is» respecte donc toutes les règles syntaxiques du basilecte sans pour autant en faire partie lexicalement.

La présence d'éléments anglais standard dans ce texte, à l'exception du lexique, s'explique par le fait que le basilecte, comme toute autre variété linguistique, s'adapte à son milieu. Du fait que les variétés créolisées sont des langues orales et non standardisées, elles sont beaucoup plus flexibles et plus susceptibles de subir ce genre d'influences environnementales.

Ce type d'hybridation linguistique constitue l'antithèse du processus d'homogénéisation que tentait d'imposer le colonisateur aux langues autochtones. Par conséquent, il n'y aurait, dans une pratique de traduction quelconque concernant un texte dans une langue métissée, ni une notion de correspondance linguistique ni une notion de correspondance textuelle pour le contenu de ce texte. En effet, ce texte s'avérerait difficilement traduisible, au sens «normal» du terme, dans une langue précise quelconque (voir Mehrez 1992: 121-122).

Jacques Derrida, qui s'intéresse aussi à la question de l'influence de l'hybridation linguistique sur la manière dont nous comprenons la traduction, explique qu'il serait impossible de transférer, dans un texte d'arrivée, l'hétérogénéité interne d'un texte de départ, écrit dans une langue métissée. Nous voudrions souligner ici ses commentaires sur le métissage linguistique de Finnegans Wake de Joyce et de Pierre Ménard de Borges. À propos de la phrase «And he war» de Joyce, il se demande: «Dans quelle langue ceci est-il écrit?» Il conclut: «malgré la multiplicité des langues, des références culturelles, des condensations, l'anglais, sans conteste, est la langue dominante». Mais, ajoute-il, «le mot allemand (war) pèse sur le mot anglais ». Commentant aussi le remaniement de Don Quixote (de Cervantes), par Borges, dans Pierre Ménard, il demande si la traduction (conventionnelle) peut tenir compte de ce texte, rédigé en espagnol, mais fortement marqué par une atmosphère française. Ainsi, déclare-t-il, : «la traduction peut tout, sauf marquer cette différence linguistique inscrite dans la langue, cette différence de système de langues inscrite dans une seule langue; à la limite elle peut tout faire passer [...] sauf le fait qu'il y a, dans un système linguistique, peut-être plusieurs langues» $(1988: 134)$. 
Cependant, les questions théoriques concernant les langues métissées en traduction dépassent de loin les problèmes d'équivalence et de fidélité. Comme ces langues sont principalement orales, elles manifestent une fluidité extraordinaire, qui aboutit à la polysémie et aux changements fréquents du sens d'un message. Un cas très précis de ce type de difficultés serait le dépôt en justice, dans le cadre de l'affaire United States v. Derrick Riley, et al, en 1998, d'enregistrements de conversations entre des membres d'un gang jamaïquain soupçonnés d'avoir commis un meurtre. Selon le linguiste Patrick et le juriste Buell, coauteurs d'un article sur les problèmes de la traduction du créole dans ce procès, la langue non-standard métissée aurait permis aux suspects d'éviter la justice pendant un certain temps. " [They] managed to escape law enforcement efforts in part by conducting their activities in J[amaican]C[reole], which appears to the average police officers to be an impenetrably dense form of an unwritten foreign language» (Patrick et Buell: 6). L'argot américain ajouté à cette langue non-standard a produit un mélange encore plus insolite. Traduire dans un tel contexte a requis un effort assidu pour construire, étape par étape, l'édifice contextuel, social et situationnel sous-jacent au contenu des conversations des suspects. On pourrait croire que cette situation est très rare, donc périphérique à l'enseignement de la traduction. Or, le métissage linguistique, souvent nié en traduction (Price 2000: 36-37), devient de plus en plus habituel dans le contexte de la mondialisation. La décomposition et la recomposition des sociétés métropolitaines urbaines, les migrations et les métissages continus qui en découlent indiquent que les variétés linguistiques issues de ce genre de contexte peuvent avoir des conséquences capitales sur la communication quotidienne.

\section{Conclusion}

Les constats que nous venons de faire nous amènent à soulever quelques questions: le contexte des langues métissées peut-il nous offrir d'autres outils de réflexion sur la traduction? Comment concevoir la traduction et comment l'enseigner de sorte qu'elle tienne compte des situations d'hybridation linguistique? Dans ce contexte, quel serait le rôle des notions comme l'équivalence et la fidélité, dont la valeur repose sur le rapport d'égalité que la normalisation ou la standardisation attribue à la langue, rapport qui a servi historiquement de modèle pour la traduction?

En ce qui concerne la formation des traducteurs, on souligne souvent l'importance de deux compétences: 1) la compétence linguistique, dont la connaissance explicite de la grammaire et la structure de la langue dans laquelle le texte à traduire est rédigé et 2) la compétence extralinguistique, c'est-à-dire la connaissance du contexte social, culturel et situationnel pertinent à l'énoncé. Il est cependant très problématique de parler de compétence linguistique relative à une «langue » quand il s'agit de variété métissée ou hybride. Les grammaires de ces langues non standard ne sont pas toujours réductibles de la même manière que celles des langues standard puisqu'elles comprennent, le plus souvent, des apports de sources diverses. Quant à la prétendue compétence extralinguistique, par contre, les langues métissées nous montrent l'importance de connaissances culturelles, sociales et situationnelles, ce qui pourrait nous amener à concevoir l'enseignement de la traduction autrement. Une nouvelle conception de la formation des traducteurs pourrait être plus axée sur les variables culturelles, sociales et situationnelles qui touchent la langue. Cela suggère 
une approche plutôt coopérative en traduction, comme celle que témoigne le travail de Patrick et Buell.

Il semble pourtant que la considération la plus pertinente pour le traitement de la langue métissée en traduction soit l'évaluation des variables du contexte cible qui conditionnent l'usage d'un texte traduit. Dans cette optique, il incombe au traducteur de connaître le contexte de réception du texte afin d'être capable de modifier son comportement conformément à ce contexte. Cette conception de la pratique remettrait en question l'idée qu'il existe une équivalence préalable entre un texte rédigé en langue $\mathrm{A}$ et un texte rédigé en langue $\mathrm{B}$ et qu’il existe un procédé défini pour la réalisation d'une traduction. Elle accorderait la primauté au contexte culturel, social et situationnel du récepteur, ce qui veut dire qu'un texte rédigé dans une variété standard n'est pas forcément traduisible dans une variété "homologue» standard et qu'une variété non standard n'est pas forcément traduisible dans une variété non standard: le traducteur ne cherche pas à transférer un sens fixe entre codes fixes mais à créer, selon les outils linguistiques d'une communauté réceptrice, des textes significatifs. Les textes d'arrivée ne seraient plus basés sur une «langue» d'arrivée quelconque, mais sur les possibilités lectales d'une communauté. La formation des traducteurs ne renverrait donc pas à la compétence en langue $\mathrm{A}$ et en langue $\mathrm{B}$, mais plutôt à la compétence à l'égard des sociolectes, des dialectes, etc., selon le contexte de leur travail. De ce fait, si la théorisation de la traduction s'élargissait pour faire une place à la langue métissée, celle-ci pourrait offrir un nouvel outil de réflexion sur la traduction, un outil plus pointu.

\section{NOTES}

1. Selon Joseph, ce qu'on appelle «standardisation» linguistique est un processus propre à l'Occident puisqu'elle dépend des institutions culturelles «which represent specific historical developments within Western civilization» (1987: 20). Des linguistes tels que Roy Harris et Daniel Baggioni font le même constat. La notion de langue standard, toujours selon Joseph, fait partie du discours linguistique d'autres sociétés à cause de l'influence du latin et des langues standardisées selon le modèle du latin. Par ailleurs, dans des cultures littéraires anciennes comme en Inde ou en Chine, la notion de langue standard ne constituait pas une catégorie analytique.

2. Suivant Baggioni (1994:28), nous entendons par normalisation, la création d'une variété relativement homogène et stable de plusieurs dialectes ou variétés. La standardisation, toujours selon Baggioni, consiste dans l'imposition de la norme sur un territoire.

3. Tiré de «Look to di pill, PJ», The Daily Gleaner, 20 mars 2001.

4. Notre traduction du texte créole. Nous tenons à signaler que cette traduction est «normative» et ne tient pas compte des problèmes inhérents au transfert entre le créole et l'anglais. Nous avons choisi de faire ce genre de traduction naïve puisque notre but est de faire parvenir au lecteur le sens de l'extrait.

5. En général, les parlers créolisés des Caraïbes sont regroupés en continuums dialectaux divisés en trois couches. D'un côté, le basilecte et de l'autre l'acrolecte et entre les deux, des variétés traitées globalement comme le mésolecte. Ces couches linguistiques reflètent plus ou moins les couches sociales établies pendant l'esclavage, qui comptait les Africains (locuteurs du basilecte), en bas de l'échelle sociale, les Européens (locuteurs de l'acrolecte/la langue standard), en haut de la même échelle, et les métis (locuteurs du mésolecte), entre les deux.

\section{RÉFÉRENCES}

AL-Sнавав, O. S. (1996): Interpretation and the Language of Translation: Creativity and Conventions in Translation, Londres, Janus Publishing Company.

BAggioni, D. (1997): Langues et nations en Europe, Paris, Payot/Rivages. 
Beaugrande, R. de (1994): «Cognition, Communication, Translation, Instruction: The Geopolitics of Discourse», dans Abdulla Shunnaq et Mohamed Heliel (dir.), Language, Discourse and Translation in the West and Middle East, Amsterdam/Philadelphia, John Benjamins, p. 1-22.

Chomsky, N. (1965): Aspects of the Theory of Syntax, Cambridge (Mass), MIT Press.

Derrida, J. (1988): "Table ronde sur la traduction», dans Claude Lévesque et Christie McDonald (dir.), L'oreille de l'autre: otobiographie, transferts, traductions, textes et débats avec Jacques Derrida, Lincoln/Londres, University of Nebraska Press, p. 124-212.

Harris, R. (1980): The Language Makers, Ithaca, New York, Cornell University Press.

Harris, R. (1981): The Language Myth, New York, St Martin's Press.

Joseph, John Earl (1987): Eloquence and Power: The Rise of Language Standards and Standard Languages, Londres, F. Pinter.

Menrez, S. (1992) : «Translation and the Postcolonial Experience: The Francophone North African Text», dans Lawrence Venuti (dir.), Rethinking Translation, London/New York, Routledge, p. $120-138$.

NidA, E. (1964): Toward a Science of Translating. Leiden, E. J. Brill.

NidA, E. et C. TABer (1974 [1969]): The Theory and Practice of Translation, ré-impression, Leiden, E. J. Brill.

Ojo, S. A. (1986): «The Role of the Translator of African Written Literature in Inter-Cultural Consciousness and Relationships", Meta 31-3, Prismes de traduction littéraire/Facets of Literary Translation, p. 291-299.

Patrick, P. et S. Buell (s.d.): «Competing Creole Transcripts on Trial» [en ligne] <http:// privatewww.essex.ac.uk/ patrickp/papers/CreoleTranscripts.pdf $>$ (page consultée le 20 mars 2003).

Pergnier, M. (1993): Les fondements sociolinguistiques de la traduction, édition remaniée, Lille, Presses universitaires de Lille.

Price, J. (2000) : «Hybrid Languages, Translation and Post-Colonial Challenges», dans Marilyn Gaddis Rose (dir.), Translation Perspectives XI: Beyond the Western Tradition, Binghampton, Centre for Research in Translation, State University of New York, p. 23-50.

Pym, A. (1992): Translation and Text Transfer: An Essay on the Principles of Intercultural Communication, Frankfurt am Main et Berlin, Peter Lang.

Reynolds, C. R. (2001) : «Look to di Pill, PJ», The Daily Gleaner [en ligne], <http://www.jamaicagleaner.com/gleaner/20010320/cleisure/cleisure4.html> (page consultée le 20 mars 2003).

Saussure, F. de (1967 [1916]) : Cours de Linguistique générale, publié par Charles Bally et Albert Sechehaye avec la collaboration de Albert Riedlinger, Paris, Payot.

Seleskovitch, D. et M. Lederer (1986): Interpréter pour traduire, $2^{\mathrm{e}}$ édition, Paris, Didier Érudition.

Snell-Hornby, M. (1995): Translation Studies: An Integrated Approach, édition révisée, Amsterdam/Philadelphia, John Benjamins.

Wright, R. (1997) : «Translation Between Latin and Romance in the Early Middle Ages», dans Jeanette Beer (dir.), Translation Theory and Practice in the Middle Ages, Kalamazoo (Michigan), Western Michigan University, p. 7-32. 